\title{
Influence of Concurrent and Adjuvant Temozolomide on Health-Related Quality of Life of Patients with Grade III Gliomas: A Secondary Analysis of a Randomized Clinical Trial (KNOG-1101 Study)
}

\author{
Grace S. Ahn', Kihwan Hwang ${ }^{1}$, Tae Min Kim², Chul Kee Park ${ }^{3}$, Jong Hee Chang', Tae-Young Jung ${ }^{5}$, Jin Hee Kim ${ }^{6}$, Do-Hyun Nam ${ }^{7}$, \\ Se-Hyuk Kim ${ }^{8}$, Heon Yoo ${ }^{9}$, Yong-Kil Hong ${ }^{10}$, Eun-Young Kim ${ }^{11}$, Dong-Eun Lee ${ }^{12}$, Jungnam Joo ${ }^{12}$, Yu Jung Kim ${ }^{13}$, Gheeyoung Choe ${ }^{14}$, \\ Byung Se Choi ${ }^{15}$, Seok-Gu Kang ${ }^{4}$, Jeong Hoon Kim ${ }^{16}$, Chae-Yong Kim ${ }^{1}$ \\ ${ }^{1}$ Department of Neurosurgery, Seoul National University Bundang Hospital, Seoul National University College of Medicine, Seongnam, \\ Departments of ${ }^{2}$ Internal Medicine and ${ }^{3}$ Neurosurgery, Seoul National University Hospital, Seoul National University College of Medicine, Seoul, \\ ${ }^{4}$ Department of Neurosurgery, Severance Hospital, Yonsei University College of Medicine, Seoul, ${ }^{5}$ Department of Neurosurgery, Chonnam National \\ University Hwasun Hospital, Hwasun, ${ }^{6}$ Department of Radiation Oncology, Keimyung University Dongsan Medical Center, Keimyung University \\ School of Medicine, Daegu, 'Department of Neurosurgery, Samsung Medical Center, Sungkyunkwan University School of Medicine, Seoul, \\ ${ }^{8}$ Department of Neurosurgery, Ajou University Hospital, Ajou University School of Medicine, Suwon, ${ }^{9}$ Department of Neuro-Oncology Clinic, \\ Center for Specific Organs Cancer, National Cancer Center Hospital, National Cancer Center, Goyang, ${ }^{10}$ Department of Neurosurgery, Seoul St. Mary's \\ Hospital, College of Medicine, The Catholic University of Korea, Seoul, ${ }^{11}$ Department of Neurosurgery, Inha University Hospital, Inha University School \\ of Medicine, Incheon, ${ }^{12}$ Division of Cancer Epidemiology and Management, Research Institute, National Cancer Center, Goyang, Departments of \\ ${ }^{13}$ Internal Medicine, ${ }^{14}$ Pathology, and ${ }^{15}$ Radiology, Seoul National University Bundang Hospital, Seoul National University College of Medicine, \\ Seongnam, ${ }^{16}$ Department of Neurological Surgery, Asan Medical Center, University of Ulsan College of Medicine, Seoul, Korea
}

Purpose The KNOG-1101 study showed improved 2-year progression-free survival (PFS) with temozolomide during and after radiotherapy compared to radiotherapy alone for patients with anaplastic gliomas. This trial investigates the effect of concurrent and adjuvant temozolomide on health-related quality of life (HRQoL).

Materials and Methods In this randomized, open-label, phase II trial, 90 patients with World Health Organization grade III glioma were enrolled across multiple centers in South Korea between March 2012 to February 2015 and followed up through 2017. The European Organization for Research and Treatment of Cancer Quality of Life Questionnaire 30 (EORTC QLQ-C30) and 20-item EORTC QLQ-Brain Neoplasm (QLQ-BN20) were used to compare HRQoL between patients assigned to concurrent chemoradiotherapy with temozolomide followed by 6 cycles of adjuvant temozolomide (arm A) and radiotherapy (RT) alone (arm B).

Results Of the 90 patients in the study, 84 patients (93.3\%) completed the baseline HRQoL questionnaire. Emotional functioning, fatigue, nausea and vomiting, dyspnea, constipation, appetite loss, diarrhea, seizures, itchy skin, drowsiness, hair loss, and bladder control were not affected by the addition of temozolomide. All other items did not differ significantly between arm A and arm B throughout treatment. Global health status particularly stayed consistent at the end of adjuvant temozolomide $(p=0.47)$ and at the end of RT $(p=0.33)$.

Conclusion The addition of concurrent and adjuvant temozolomide did not show negative influence on HRQoL with improvement of PFS for patients with anaplastic gliomas. The absence of systematic and clinically relevant changes in HRQoL suggests that an overall long-term net clinical benefit exists for concurrent and adjuvant temozolomide.

Key words Quality of life, Anaplastic glioma, 1p/19q co-deletion, Temozolomide, Chemotherapy

\section{Introduction}

Anaplastic gliomas account for $20 \%$ of adult gliomas which comprise the most common type of primary brain tumors [1]. The current standard of glioma management has been to prioritize overall and progress-free survival by focusing on surgery followed by radiotherapy (RT) [2]. There is growing evidence of increased survival with the advent of temozolomide in combination with RT in glioblastomas [3-5]. However, the overall advantage of combined chemotherapy with conventional cytotoxic agents for World Health Organization (WHO) grade III gliomas remains controversial [1]. For 1p/19q-co-deleted gliomas, PCV (procarbazine, lomustine, and vincristine) is proven to be beneficial. According to the 
CATNON interim results, adjuvant temozolomide is proven to be associated with improved survival [6]. The point yet to be elucidated is the efficacy of concurrent RT with temozolomide for 1p/19q-non-co-deleted gliomas [1,7-9].

Molecular markers are increasingly utilized not only as diagnostic markers but also as prognostic and predictive factors for patient response and chemoradiosensitivity [10]. Such molecular markers in glioma management include co-deletion of chromosome $1 \mathrm{p} / 19 \mathrm{q}$, methylation status of $\mathrm{O}^{6}$-methylguanine-DNA methyltransferase promoter, and isocitrate dehydrogenase (IDH) mutation [3,11-13], among which IDH mutation remains the most significant marker of survival in WHO grade III gliomas. Another indicator of prognosis in addition to IDH mutation, the $1 \mathrm{p} / 19 \mathrm{q}$ chromosome delineates prognosis in clinically low-grade gliomas [14]. These $1 \mathrm{p} / 19 \mathrm{q}$ non-co-deleted gliomas may experience worse prognosis and faster tumor growth requiring further attention. For example, patients with $1 \mathrm{p} / 19 \mathrm{q}$ co-deleted gliomas have been shown to benefit more from adjuvant PCV compared with those with $1 \mathrm{p} / 19 \mathrm{q}$ non-co-deleted gliomas [15]. However, adjuvant temozolomide benefits $1 p / 19 q$ nonco-deleted gliomas [6], while early chemotherapy has been shown to benefit anaplastic diffuse gliomas regardless of $1 \mathrm{p} / 19 \mathrm{q}$ status [1].

As evidence grows for combined chemoradiotherapy for gliomas with the advent of temozolomide, it is critical to investigate the net clinical benefit of each new treatment modality. While optimal indications, dosing protocols and survival analysis are all needed to review and standardize regimen of care, consideration of a patient's overall physical, social, and psychological well-being during treatment is imperative. Possible benefits of a new treatment, in terms of prolonged survival, have yet to be carefully weighed against potential negative effects of treatment on the patients' quality of life. Accordingly, the focus of low-grade glioma management has been to prolong the disease-free state often in place of improving overall health-related quality of life (HRQoL) during the critical treatment period [16]. The risk of deterioration in physical, social, and psychological well-being in altering regimen of care can lead to decreased quality of life for glioma patients and should be heavily considered during treatment improvement and standardization.

The KNOG-1101 study has proven improved 2-year progression-free survival (PFS) with the addition of concurrent and adjuvant temozolomide for patients with anaplastic glioma. However, even with improved PFS outcomes, concurrent and adjuvant temozolomide is not wholly curative of total disease. Any improvement in cancer progression must be carefully weighed against the potential negative treatment effects on the patients' independence and quality of life. Concerns regarding the influence of additional chemotherapy on patients' HRQoL have therefore been raised. This secondary analysis of KNOG-1101, a randomized open-label phase II study, evaluates the difference in HRQoL between patients treated with RT alone versus combined RT and chemotherapy. Herein, we report on the influence of treatment combining RT with concurrent and adjuvant chemotherapy on patients' HRQoL which was a predefined secondary objective of the randomized clinical trial.

\section{Materials and Methods}

\section{Study population}

Patients with newly diagnosed histologically proven supratentorial anaplastic gliomas (WHO grade III) without $1 p / 19 q$ co-deletion were eligible to participate in this study. Inclusion criteria were as follows: diagnosis of anaplastic gliomas according to 2007 WHO criteria, age $\geq 18$ years, Eastern Cooperative Oncology Group performance status of 0 or 1, stable or decreasing dose of steroids for $\geq 5$ days prior to randomization, RPA classification of III, IV or V, adequate hematologic, renal and hepatic function including an absolute neutrophil count $>1,500 / \mu \mathrm{L}$, platelet count of $\geq 100,000 / \mu \mathrm{L}$, serum creatinine level $<1.7 \mathrm{mg} / \mathrm{dL}$, bilirubin level $\leq 2.0 \mathrm{mg} / \mathrm{dL}$, and aspartate aminotransferase/alanine aminotransferase level $\leq 2.5 \times$ upper limit of the normal range at each institution.

Exclusion criteria consisted of prior chemotherapy within the last 5 years, prior RT of the head and neck area, receiving concurrent investigational agents or having received an investigational agent within 30 days prior to randomization, planned surgery for other diseases, history of malignancy with the exception of cervical carcinoma in situ or basal cell carcinoma of the skin, pregnancy or lactation, refusal to use effective contraception, concurrent illness that would interfere with the prescribed treatment and inability to undergo gadolinium-based magnetic resonance imaging (MRI).

\section{Study design and treatment}

The KOREANA study was a multicenter study performed by the Korean Neuro-Oncology Group [17]. The trial was a randomized, open-label, phase II study created to evaluate the efficacy between combination therapy with RT and temozolomide versus RT only in Korean adult patients with newly diagnosed supratentorial grade III gliomas without $1 \mathrm{p} / 19 \mathrm{q}$ co-deletion. The study protocol was registered at clinicaltrials.gov (NCT01534845).

Upon enrollment into the study, patients were diagnosed and confirmed to have anaplastic glioma at each institution. Tumor material was then assessed for $1 \mathrm{p} / 19 \mathrm{q}$ co-deletion status using fluorescent in-situ hybridization and reviewed 


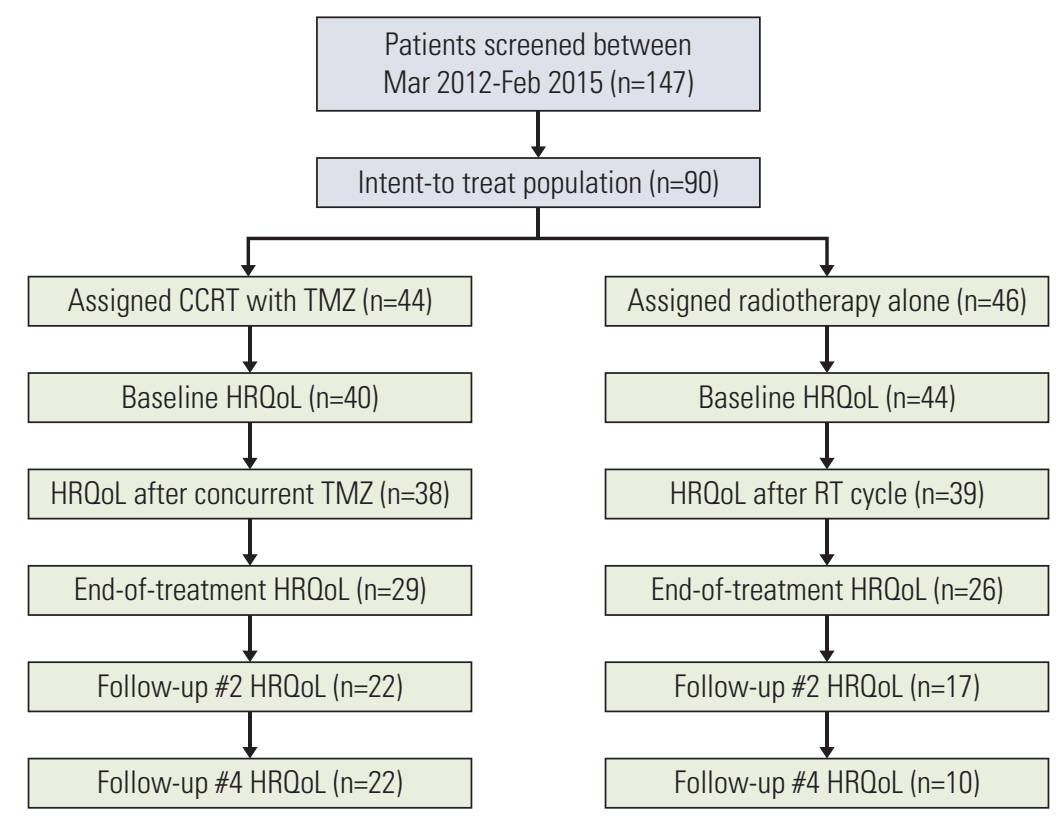

Fig. 1. Recruitment and inclusion of patients in the study (CONSORT diagram). Data includes the number of patients in each category (baseline, alive, and progression-free) who completed the HRQoL questionnaire. CCRT, concurrent chemoradiotherapy; HRQoL, healthrelated quality-of-life; RT, radiotherapy; TMZ, temozolomide.

by a pathologist (C.G.Y.) [18]. After confirmation of $1 p / 19 q$ co-deletion, patients were screened for inclusion and randomly assigned in equal numbers to receive either RT alone or RT with concurrent and adjuvant temozolomide. Clinical data were collected and validated using a web-based clinical research management platform (Velos Inc., Fremont, CA).

All patients received fractionated focal irradiation in daily fractions of 2 Gy given 5 days per week for 6 weeks for a total of $60 \mathrm{~Gy}$. When given concurrently with RT, temozolomide was administered daily from the first to the last day of RT, including on non-RT weekend days, at a dose of $75 \mathrm{mg} / \mathrm{m}^{2}$ for a maximum of 6 weeks. During adjuvant chemotherapy, temozolomide administration began 4 weeks after completion of RT for a maximum of 6 cycles. Patients received $150 \mathrm{mg} / \mathrm{m}^{2}$ temozolomide on days $1-5$ of the first cycle and $200 \mathrm{mg} / \mathrm{m}^{2}$ on days $1-5$ of subsequent cycles if no or minor toxicity was seen during the first cycle. Temozolomide was provided to individuals free of charge by MSD Korea. Upon disease progression, a rescue regimen was suggested for patients with RT-only group.

Patients were followed up weekly during RT, every 2 weeks during adjuvant temozolomide treatment, and every 3 or 4 months after completion of all treatment. Tumors were assessed with MRI 4 weeks after the end of RT, every 3 months until the second year, and every 6 months thereafter until disease progression. Radiographic response was compared to the tumor measurement obtained at pretreatment baseline measurements as a reference. Response was then categorized into four groups using new criteria proposed by the Response Assessment in Neuro-Oncology (RANO) working group. Patients with possible pseudo-progression were kept on current treatment and re-evaluated 4 weeks thereafter to clarify treatment response. Steroid dose was reviewed at every outpatient visit. Clinical evaluation with adverse event was scored with the National Cancer Institute Common Toxicity Criteria for Adverse Event ver. 4.0. European Organization for Research and Treatment of Cancer (EORTC) Quality of Life Questionnaire 30 (QLQ-C30) and 20-item EORTC QLQ-Brain Neoplasm (QLQ-BN20) were used to evaluate HRQoL at baseline and during all visits requiring an MRI scan.

\section{HRQoL assessment}

Evaluation of HRQoL was performed using the validated EORTC QLQ-C30 and QLQ-BN20. Paper questionnaires were completed at baseline and at each visit requiring an MRI. With a baseline study prior to treatment as reference, this study compares HRQoL after concurrent chemoradiotherapy (CCRT) completion (arm A) versus after RT cycle 1 without chemotherapy (arm B). In addition to global health status, five items were scored within a functional scale (physical functioning, role functioning, emotional functioning, cognitive functioning, social functioning), nine items within the symptom scale (fatigue, nausea and vomiting, pain, dysp- 
Table 1. Baseline demographic and disease characteristics

\begin{tabular}{|c|c|c|c|c|}
\hline & $\begin{array}{l}\text { Total } \\
(n=84)\end{array}$ & $\begin{array}{c}\text { CCRT with } \\
\text { temozolomide } \\
\text { plus adjuvant } \\
\text { temozolomide }(n=40)\end{array}$ & $\begin{array}{l}\text { Radiotherapy } \\
\text { alone }(n=44)\end{array}$ & p-value \\
\hline Age (yr) & $44.7 \pm 13.2$ & $44.7 \pm 13.3$ & $44.8 \pm 13.2$ & $0.980^{\mathrm{a})}$ \\
\hline \multicolumn{5}{|l|}{ Sex } \\
\hline Male & $46(54.8)$ & $23(57.5)$ & $23(52.3)$ & $0.631^{\mathrm{b})}$ \\
\hline Female & $38(45.2)$ & $17(42.5)$ & $21(47.7)$ & \\
\hline \multicolumn{5}{|l|}{ ECOG PS } \\
\hline 0 & $34(40.5)$ & $12(30.0)$ & $22(50.0)$ & $0.143^{c)}$ \\
\hline 1 & $44(52.4)$ & $24(60.0)$ & $20(45.5)$ & \\
\hline 2 & $6(7.14)$ & $4(10.0)$ & $2(4.5)$ & \\
\hline \multicolumn{5}{|l|}{ Extent of tumor resection } \\
\hline Gross total resection & $37(44.1)$ & $17(42.5)$ & $20(45.5)$ & $0.681^{\mathrm{b})}$ \\
\hline Subtotal resection & $17(20.2)$ & $9(22.5)$ & $8(18.2)$ & \\
\hline Partial resection & $14(16.7)$ & $5(12.5)$ & $9(20.5)$ & \\
\hline Biopsy only & $16(19.1)$ & $9(22.5)$ & $7(15.9)$ & \\
\hline \multicolumn{5}{|c|}{ MGMT promotor methylation } \\
\hline Unmethylation & $42(64.6)$ & $21(65.6)$ & $21(63.6)$ & $0.867^{\mathrm{b})}$ \\
\hline Methylation & $23(35.4)$ & $11(34.4)$ & $12(36.4)$ & \\
\hline Missing & 19 & 8 & 11 & \\
\hline \multicolumn{5}{|l|}{ IDH1 mutation } \\
\hline Wild type & $44(62.9)$ & $22(64.7)$ & $22(61.1)$ & $0.756^{\mathrm{b})}$ \\
\hline Mutant & $26(37.1)$ & $12(35.3)$ & $14(38.9)$ & \\
\hline Missing & 14 & 6 & 8 & \\
\hline Follow-up duration (mo) & $44.9(2.8-71.1)$ & $52.9(3.6-69.7)$ & $42.1(2.8-71.1)$ & $0.209^{\mathrm{d})}$ \\
\hline
\end{tabular}

Values are presented as mean $\pm \mathrm{SD}$, number $(\%)$, or median (range). CCRT, concurrent chemoradiotherapy; ECOG PS, Eastern Cooperative Oncology Group performance status; IDH1, isocitrate dehydrogenase 1; MGMT, O6-methylguanine-DNA methyltransferase; SD, standard deviation. ${ }^{\mathrm{a})}$ Student's $\mathrm{t}$ test, ${ }^{\mathrm{b})} \mathrm{Chi}$-square test, ${ }^{\mathrm{c}}$ Fisher exact test.

nea, insomnia, appetite loss, constipation, diarrhea, financial difficulties), and 11 items within the brain module (future uncertainty, visual disorder, motor dysfunction, communication deficit, headaches, seizures, drowsiness, itchy skin, hair loss, weakness of legs, bladder control). We hypothesized that the addition of chemotherapy would result in an equivocal quality of life alongside overall improved well-being and PFS compared to RT-only treatment.

\section{Statistical methods}

Baseline characteristics, details of treatments, and adverse events were expressed as mean with standard deviation for continuous variables and frequency with percentage for categorical variables. The comparison between CCRT with temozolomide and RT only groups were carried out using Student's t test, Pearson's chi-square, or Fisher exact test, as appropriate. Results were considered statistically significant if $\mathrm{p}$-value was less than 0.05 . Statistical analyses were performed using SAS ver. 9.4 (SAS Institute Inc., Cary, NC).

\section{Results}

\section{Study participants}

Of the 147 patients screened between March 2012 and February 2015,90 patients were randomly assigned in a 1:1 ratio to receive either CCRT plus adjuvant temozolomide $(n=44)$ or RT alone $(\mathrm{n}=46)$. A total of 84 patients $(93.3 \%)$ completed at least $1 \mathrm{HRQoL}$ questionnaire at baseline: $40(90.9 \%)$ of those in the CCRT plus adjuvant temozolomide arm and 44 (95.7\%) patients in the RT alone arm (Fig. 1). The baseline demographics of the patients who provided HRQoL data were comparable to those of the intent-to-treat population and were well-balanced between treatment arms (Table 1).

\section{HRQoL completion rates and baseline scores}

Adherence to HRQoL assessments decreased from 93.3\% at baseline to $85.6 \%$ (77 of the 90 original treated patients) during treatment and dropped to $61.1 \%$ (55 of the 90 original patients) at the end of treatment (Fig. 1). Mean and median 

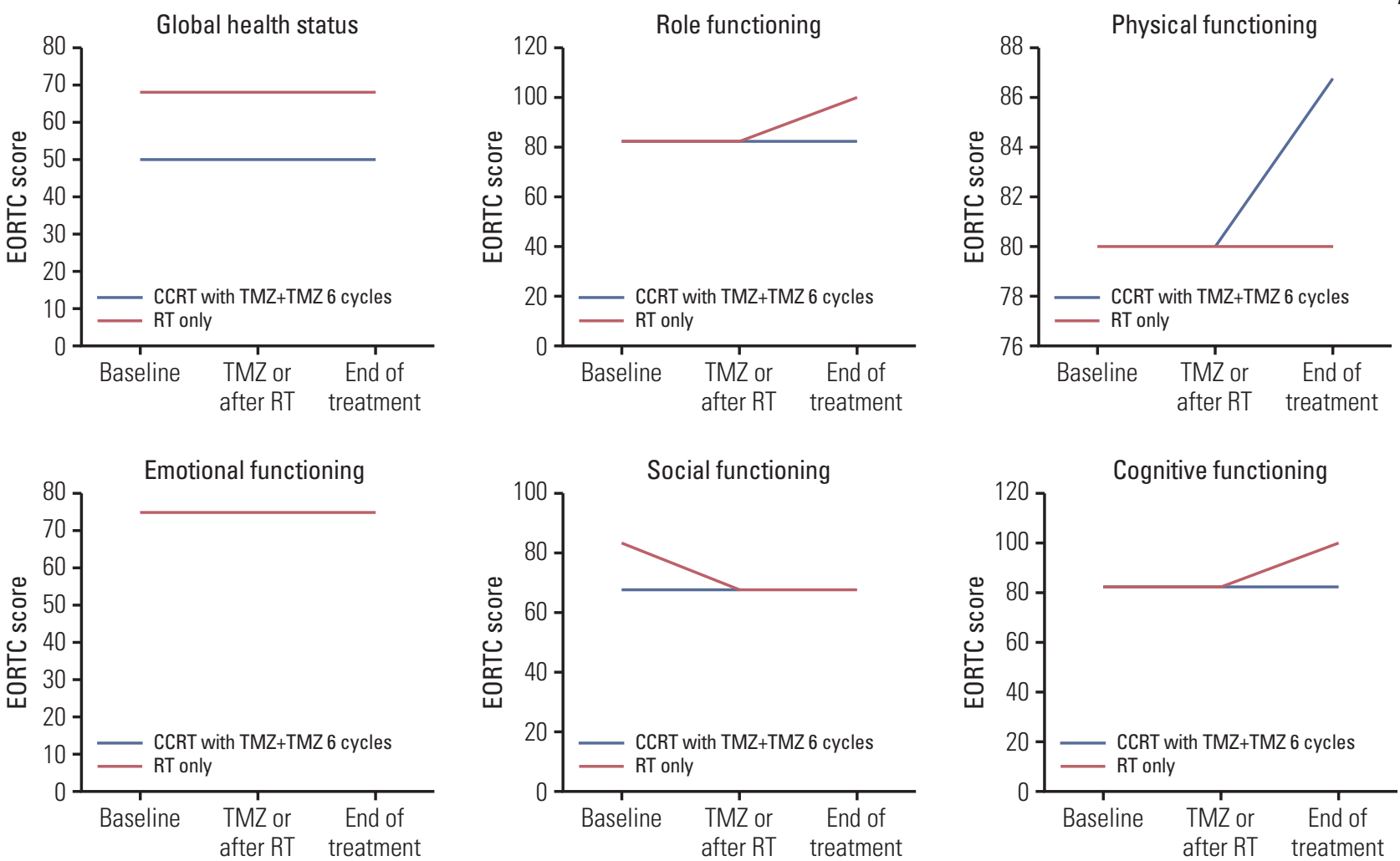

Fig. 2. (A) Stable health-related quality of life (European Organization for Research and Treatment of Cancer [EORTC] Functional Items) during treatment. High score on functional scale indicates healthy level of functioning. Baseline refers to the magnetic resonance imaging (MRI) visit before concurrent chemoradiotherapy (CCRT) or radiotherapy (RT) only begins. Temozolomide (TMZ) or after RT refers to the MRI visit either after CCRT with temozolomide or after radiotherapy alone. End of treatment refers to the MRI visit after the 6 cycles of adjuvant temozolomide or after 6 months since the end of radiotherapy alone. (Continued to the next page)

baseline HRQoL scores were comparable between arms for nearly all items (S1 Table). At baseline, the CCRT group had similar EORTC functioning scores across all scales including physical, role, emotional, cognitive while social functioning was $15.6 \%$ lower in the CCRT group. Symptom scales were similar between both groups except insomnia was apparent and financial difficulties were slightly lower in the CCRT group prior to treatment. Within the EORTC QLQ-BN20 scores at baseline, the CCRT group had slightly higher motor dysfunction, communication deficit, headaches relative to the RT-only group. While the RT-only group only had greater visual disorder scores, all other scores were similar.

\section{Stable HRQoL during progression-free time}

Mean changes in HRQoL over time for the global health status and functional scale items are presented in Fig. 2A and for the symptomatic and brain module items in Fig. 2B. Throughout the 10-month assessment period, mean changes from baseline were stable for all HRQoL scales $(<20$-point change from baseline/no significant $\mathrm{p}$-value difference) for all HRQoL items in both treatment arms.

Compared with baseline, both patients in the CCRT group and the RT-only group reported stable, unchanged scores for global health status throughout the treatment period. Both treatment arms also reported stable, similar scores for emotional functioning, fatigue, nausea and vomiting, dyspnea, constipation, appetite loss, diarrhea, seizures, itchy skin, drowsiness, hair loss and bladder control. These items across the functional, symptomatic, and brain module scale all showed equal EORTC scores beginning from baseline and did not change throughout the 10-month assessment period while receiving both treatments (S2 and S3 Tables).

\section{Mean changes in HRQoL from baseline}

Compared with baseline, the CCRT group showed improved physical functioning, insomnia, pain, perception of financial difficulties, and communication deficits throughout the 10-month assessment period. In contrast, the RT-only 

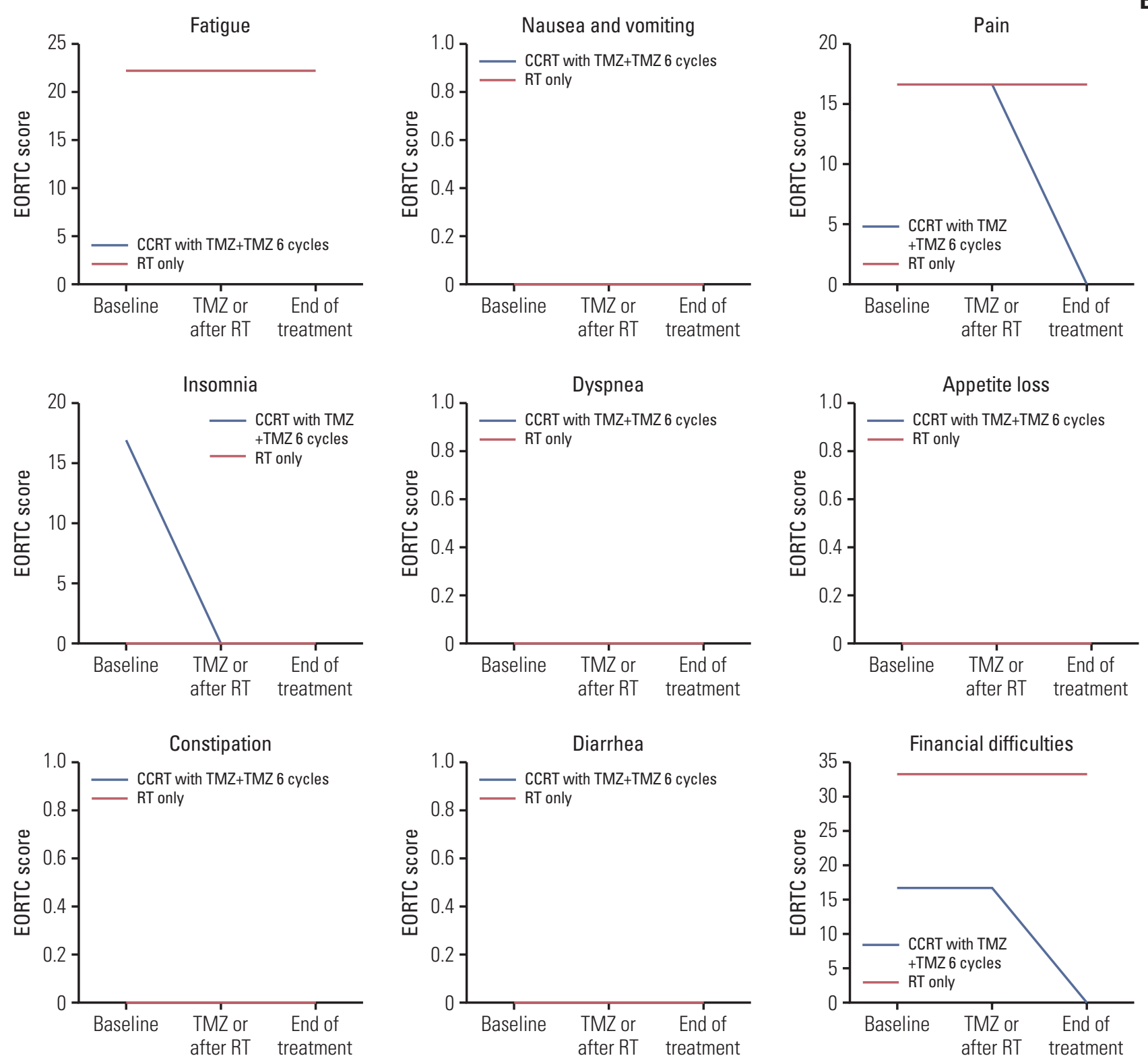

Fig. 2. (Continued from the previous page) (B) Stable health-related quality of life (EORTC Symptom and Brain Module Items) during progression-free time. High score on symptomatic and brain module scale indicates problematic levels. (Continued to the next page)

group showed slightly improved role and cognitive functioning while social functioning and headaches worsened throughout the treatment period compared with baseline. However, there were no significant differences between the CCRT and RT-only arm overall to support concluding that the CCRT group showed no adverse HRQoL outcomes relative to the RT-only group. Symptomatic scale items such as motor dysfunction and weakness of legs improved for both treatment arms compared to baseline.

Several HRQoL items such as global health status showed a weaker score for the CCRT plus adjuvant temozolomide arm. However, these scores initially differed before CCRT administration and is not a reflection of the effect of adding temozolomide to treatment.

\section{Discussion}

Anaplastic gliomas, referred to in this study as grade III gliomas via the 2007 WHO classification, account for $20 \%$ 

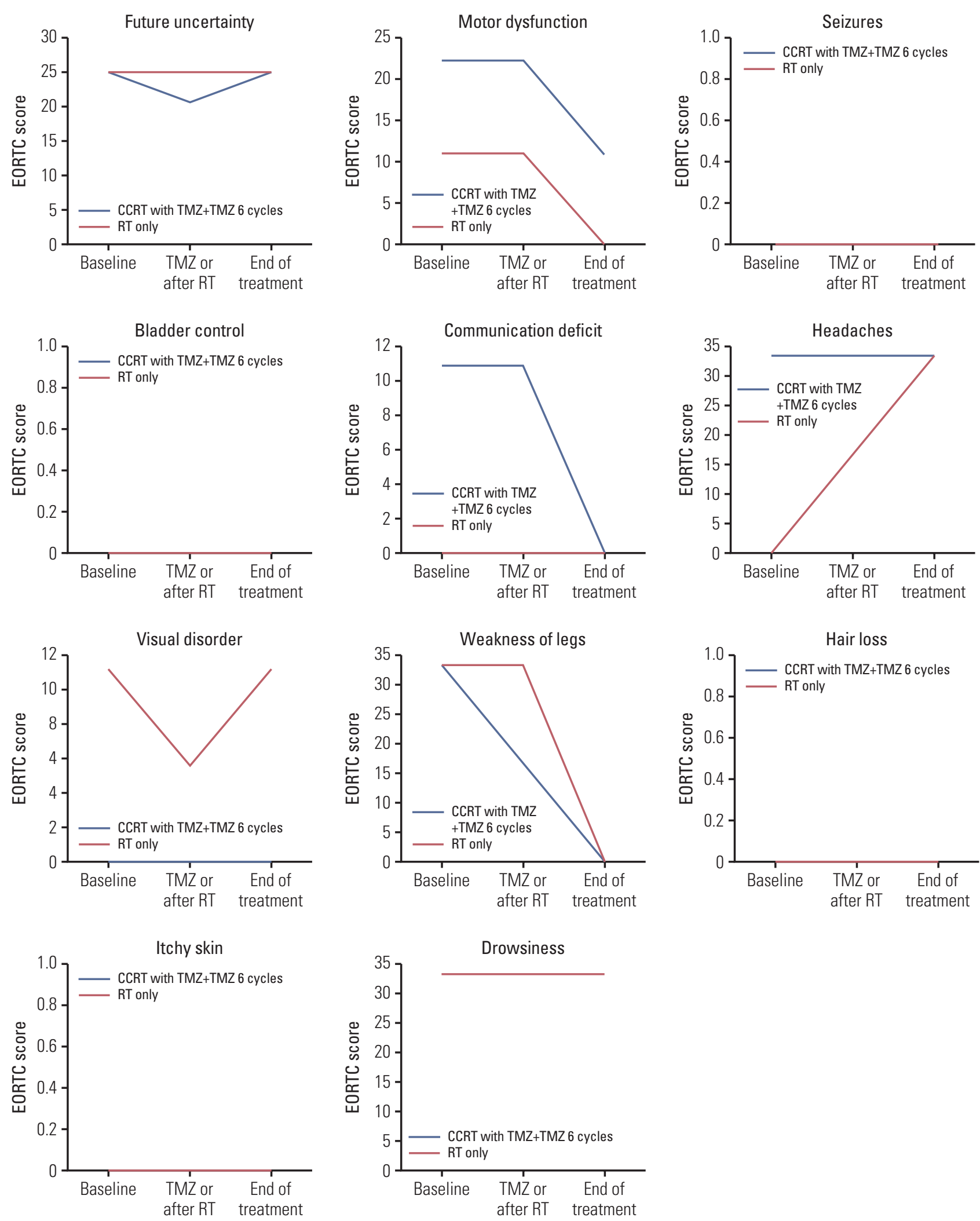

Fig. 2. (Continued from the previous page) 
of adult gliomas comprising the most common type of primary brain tumors [1]. With current management focusing on maximal surgical resection followed by adjuvant RT [2,19-21], prognosis remains poor with a median survival range of 2-5 years [22,23]. However, growing evidence for nitrosourea-based chemotherapy with the advent of temozolomide highlights the need for investigation on the effects of combined chemoradiotherapy on not only survival outcome but, more importantly, quality of life during those additional years of life [3-5,24]. The KNOG 1101 study found that temozolomide addition in Korean adult patients with newly diagnosed WHO grade III gliomas showed an improved 2-year PFS. Our study further found that not only did concurrent and adjuvant temozolomide improves 2-year PFS but also produced comparable HRQoL compared to RT only.

Our study focuses primarily on the potential effects on quality of life by adding temozolomide to current standard treatment of grade III gliomas. Although there is an initial difference in global health status between patient groups, the difference is not statistically significant and the score remains stable throughout treatment. The score difference also existed before administration of temozolomide began. Therefore, the slight difference does not reflect the effect of adding temozolomide on the HRQoL of patients with anaplastic gliomas.

There is initially no difference in cognitive functioning between patient groups, but patients with RT only were found to slightly increase in cognitive functioning. However, the difference is not statistically significant. This difference also may not necessarily be explained by the absence of temozolomide but rather secondary to the decreased sample size within the RT-only treatment arm. The response rate of RT-only patients in the HRQoL analysis decreased from an initial 44 patients who completed the baseline HRQoL down to 26 patients by the end of treatment. The death of patients throughout the 10-month assessment period decreased the number of healthier patients with better prognosis which may explain the difference in HRQoL scores by the end of treatment.

Studies continue to show the efficacy of temozolomide as shown by proven survival gain in glioblastoma as well as glioma management [25,26]. Although former studies show gastrointestinal and hematologic toxicity occasionally associated with the addition of chemotherapy, the KNOG-1101 study and this secondary analysis help solidify the argument for temozolomide with only a minimal decrease in QoL. In our analysis of HRQoL during the early 10-month treatment course between concurrent and adjuvant temozolomide with RT compared to RT alone, there is overall no significant difference or change in HRQoL score across all items. Although former studies have shown that temozolomide was occasionally associated with gastrointestinal complaints such as nausea (44\%), vomiting (37\%), an rarely leukopenia (1\%), no mortality was reported due to temozolomide [27].

The addition of other chemotherapeutics such as PCV to standard RT alone for higher grade gliomas result in improved survival. However, these chemotherapeutics were found to be more toxic compared to the addition of temozolomide as reflected in the HRQoL score comparisons in prior studies [28]. The addition of temozolomide to standard glioma treatment results in improved PFS as shown by the KNOG-1101 study and without a significant negative influence on HRQoL as shown by our analysis. Thus, combined CCRT with temozolomide is a safe and effective treatment protocol for patients diagnosed with WHO grade III gliomas and should be considered in improving standard brain tumor management for patients with anaplastic gliomas.

This study has several limitations. First, the current trial was open-label because RT alone is considered standard treatment and this alternative to temozolomide inclusion was not considered ethically unacceptable exposure to patients with glioma diagnosis which patients understood upon acceptance into the trial. Although a "placebo effect" may affect subjective end points like quality of life or even PFS by influencing the frequency of imaging and its interpretation, in the current trial a consistent benefit was observed in PFS as assessed by blinded central radiology review. Second, 63 patients were excluded from the original 147 patients during interim analysis due to lack of consent, refusal, disease progression among other reasons. Because of our smaller sample size due to initial recruitment and patient dropout due to various reasons out of our control, further studies addressing the HRQoL of temozolomide addition may be required in a larger patient population. Third, the failure to follow-up throughout the treatment period due to death or progression decreased the response rate and may have skewed the HRQoL scores between groups by the end of treatment.

\section{Electronic Supplementary Material}

Supplementary materials are available at Cancer Research and Treatment website (https:// www.e-crt.org).

\section{Ethical Statement}

A total of 14 Korean institutions participated in this study with an ethics approval from their respective institutional review boards before enrollment began. All patients provided written informed consent according to national guidelines. No patients were compensated for their participation in this study.

\section{Author Contributions}

Conceived and designed the analysis: Kim CY.

Collected the data: Kim TM, Park CK, Chang JH, Jung TY, Kim JH, Nam DH, Kim SH, Yoo H, Hong YK, Kim EY, Lee DE, Joo J, Kim YJ, 
Choe G, Choi BS, Kang SG, Kim JH, Kim CY.

Contributed data or analysis tools: Ahn GS, Hwang K, Lee DE, Joo J. Performed the analysis: Ahn GS, Hwang K, Lee DE, Joo J.

Wrote the paper: Ahn GS, Hwang K.

\section{Conflicts of Interest}

Conflict of interest relevant to this article was not reported.

\section{Acknowledgments}

This study was supported by a grant from the National R\&D Program for Cancer Control, Ministry of Health and Welfare, Republic of Korea (1120320), and was partly supported by grant no. 02-2018018 from the Seoul National University Bundang Hospital Research Fund.

\section{References}

1. Izquierdo C, Joubert B, Ducray F. Anaplastic gliomas in adults: an update. Curr Opin Oncol. 2017;29:434-42.

2. Siker ML, Chakravarti A, Mehta MP. Should concomitant and adjuvant treatment with temozolomide be used as standard therapy in patients with anaplastic glioma? Crit Rev Oncol Hematol. 2006;60:99-111.

3. Hegi ME, Diserens AC, Gorlia T, Hamou MF, de Tribolet $\mathrm{N}$, Weller M, et al. MGMT gene silencing and benefit from temozolomide in glioblastoma. N Engl J Med. 2005;352:9971003.

4. Stupp R, Hegi ME, Mason WP, van den Bent MJ, Taphoorn MJ, Janzer RC, et al. Effects of radiotherapy with concomitant and adjuvant temozolomide versus radiotherapy alone on survival in glioblastoma in a randomised phase III study: 5-year analysis of the EORTC-NCIC trial. Lancet Oncol. 2009;10:45966.

5. Stupp R, Mason WP, van den Bent MJ, Weller M, Fisher B, Taphoorn MJ, et al. Radiotherapy plus concomitant and adjuvant temozolomide for glioblastoma. N Engl J Med. 2005;352:987-96.

6. van den Bent MJ, Baumert B, Erridge SC, Vogelbaum MA, Nowak AK, Sanson M, et al. Interim results from the CATNON trial (EORTC study 26053-22054) of treatment with concurrent and adjuvant temozolomide for $1 \mathrm{p} / 19 \mathrm{q}$ nonco-deleted anaplastic glioma: a phase 3, randomised, openlabel intergroup study. Lancet. 2017;390:1645-53.

7. Kim YH, Park CK, Cho WH, Kim IA, Moon S, Choe G, et al. Temozolomide during and after radiation therapy for WHO grade III gliomas: preliminary report of a prospective multicenter study. J Neurooncol. 2011;103:503-12.

8. Vogelbaum MA, Hu C, Peereboom DM, Macdonald DR, Giannini C, Suh JH, et al. Phase II trial of pre-irradiation and concurrent temozolomide in patients with newly diagnosed anaplastic oligodendrogliomas and mixed anaplastic oligoastrocytomas: long term results of RTOG BR0131. J Neurooncol. 2015;124:413-20.

9. Berghoff A, van den Bent M. How I treat anaplastic glioma without 1p/19q codeletion. ESMO Open. 2019;4:e000534.

10. Cairncross JG, Ueki K, Zlatescu MC, Lisle DK, Finkelstein DM, Hammond RR, et al. Specific genetic predictors of chemotherapeutic response and survival in patients with anaplastic oligodendrogliomas. J Natl Cancer Inst. 1998;90:1473-9.

11. Hartmann C, Hentschel B, Wick W, Capper D, Felsberg J,
Simon M, et al. Patients with IDH1 wild type anaplastic astrocytomas exhibit worse prognosis than IDH1-mutated glioblastomas, and IDH1 mutation status accounts for the unfavorable prognostic effect of higher age: implications for classification of gliomas. Acta Neuropathol. 2010;120:707-18.

12. Kamiryo T, Tada K, Shiraishi S, Shinojima N, Kochi M, Ushio Y. Correlation between promoter hypermethylation of the O6-methylguanine-deoxyribonucleic acid methyltransferase gene and prognosis in patients with high-grade astrocytic tumors treated with surgery, radiotherapy, and 1-(4-amino2-methyl-5-pyrimidinyl)methyl-3-(2-chloroethyl)-3-nitrosourea-based chemotherapy. Neurosurgery. 2004;54:349-57.

13. Smith JS, Perry A, Borell TJ, Lee HK, O’Fallon J, Hosek SM, et al. Alterations of chromosome arms $1 p$ and $19 q$ as predictors of survival in oligodendrogliomas, astrocytomas, and mixed oligoastrocy tomas. J Clin Oncol. 2000;18:636-45.

14. Cairncross G, Wang M, Shaw E, Jenkins R, Brachman D, Buckner J, et al. Phase III trial of chemoradiotherapy for anaplastic oligodendroglioma: long-term results of RTOG 9402. J Clin Oncol. 2013;31:337-43.

15. van den Bent MJ, Brandes AA, Taphoorn MJ, Kros JM, Kouwenhoven MC, Delattre JY, et al. Adjuvant procarbazine, lomustine, and vincristine chemotherapy in newly diagnosed anaplastic oligodendroglioma: long-term follow-up of EORTC brain tumor group study 26951. J Clin Oncol. 2013;31:344-50.

16. Kim CW, Joo JD, Kim YH, Han JH, Kim CY. Health-related quality of life in brain tumor patients treated with surgery: preliminary result of a single institution. Brain Tumor Res Treat. 2016;4:87-93.

17. Hwang K, Kim TM, Park CK, Chang JH, Jung TY, Kim JH, et al. Concurrent and adjuvant temozolomide for newly diagnosed grade III gliomas without $1 p / 19 q$ co-deletion: a randomized, open-label, phase 2 study (KNOG-1101 Study). Cancer Res Treat. 2020;52:505-15.

18. Jenkins RB, Curran W, Scott CB, Cairncross G. Pilot evaluation of $1 p$ and $19 q$ deletions in anaplastic oligodendrogliomas collected by a national cooperative cancer treatment group. Am J Clin Oncol. 2001;24:506-8.

19. Walker MD, Alexander E Jr, Hunt WE, MacCarty CS, Mahaley Ms Jr, Mealey J Jr, et al. Evaluation of BCNU and/or radiotherapy in the treatment of anaplastic gliomas: a cooperative clinical trial. J Neurosurg. 1978;49:333-43.

20. Walker MD, Green SB, Byar DP, Alexander E Jr, Batzdorf U, 
Brooks WH, et al. Randomized comparisons of radiotherapy and nitrosoureas for the treatment of malignant glioma after surgery. N Engl J Med. 1980;303:1323-9.

21. DeAngelis LM. Brain tumors. N Engl J Med. 2001;344:114-23.

22. Simonetti G, Gaviani P, Innocenti A, Botturi A, Lamperti E, Silvani A. Update on treatment strategies for anaplastic glioma: a review of literature. Neurol Sci. 2014;35:977-81.

23. Sasaki H, Yoshida K. Treatment recommendations for adult patients with diffuse gliomas of grades II and III according to the new WHO classification in 2016. Neurol Med Chir (Tokyo). 2017;57:658-66.

24. Chibbaro S, Benvenuti L, Caprio A, Carnesecchi S, Pulera F, Faggionato F, et al. Temozolomide as first-line agent in treating high-grade gliomas: phase II study. J Neurooncol. 2004;67:77-81.
25. Joo JD, Chang JH, Kim JH, Hong YK, Kim YH, Kim CY. Temozolomide during and after radiotherapy for newly diagnosed glioblastomas: a prospective multicenter study of Korean patients. J Korean Neurosurg Soc. 2012;52:92-7.

26. Joo JD, Kim H, Kim YH, Han JH, Kim CY. Validation of the effectiveness and safety of temozolomide during and after radiotherapy for newly diagnosed glioblastomas: 10-year experience of a single institution. J Korean Med Sci. 2015;30: 1597-603.

27. Bae SH, Park MJ, Lee MM, Kim TM, Lee SH, Cho SY, et al. Toxicity profile of temozolomide in the treatment of 300 malignant glioma patients in Korea. J Korean Med Sci. 2014;29:980-4.

28. Dirven L, Aaronson NK, Heimans JJ, Taphoorn MJ. Healthrelated quality of life in high-grade glioma patients. Chin J Cancer. 2014;33:40-5. 